\title{
Effects of Surface Charge on the Fate and Phytotoxicity of Gold Nanoparticles to Phaseolus vulgaris
}

\author{
Xingmao $\mathrm{Ma}^{{ }^{*}}$ and Bryan Quah ${ }^{2}$ \\ ${ }^{1}$ Zachry Department of Civil Engineering, Texas A\&M University, 3136 TAMU College Station, TX, 77843, USA \\ ${ }^{2}$ Department of Civil and Environmental Engineering, Southern Illinois University Carbondale, Carbondale, IL, 62901, USA
}

"Correspondence to:

Xingmao Ma

Zachry Department of Civil Engineering Texas A\&M University 3136 TAMU College Station TX, 77843, USA

Tel: 979-862-1772

E-mail:xma@civil.tamu.edu

Received: December 11, 2015

Accepted: February 26, 2016

Published: March 04, 2016

Citation: Ma X, Quah B. 2016. Effects of Surface Charge on the Fate and Phytotoxicity of Gold Nanoparticles to Phaseolus vulgaris. J Food Chem Nanotechnol 2(1): 57-65.

Copyright: (C) 2016 Ma and Quah. This is an Open Access article distributed under the terms of the Creative Commons Attribution 4.0 International License (CC-BY) (http://creativecommons. org/licenses/by/4.0/) which permits commercial use, including reproduction, adaptation, and distribution of the article provided the original author and source are credited.

Published by United Scientific Group

\begin{abstract}
Rapid advancement of nanotechnology has caused serious concerns over the potential release and accumulation of engineered nanoparticles (ENPs) in the environment. Gold nanoparticles (AuNPs) in particular, has attracted significant attention due to their broad applications. However, most previous toxicity studies have been heavily focused on animal and human cell lines, with limited information available for the AuNPs plant interactions. This study aimed to elucidate the impact of surface charge on the phytotoxicity and plant uptake of AuNPs by Phaseolus vulgaris (bush bean), an important food crop. The results showed that $5 \mathrm{mg} / \mathrm{L}$ of AuNPs exerted inconsequential impact on the physiological processes of plants, regardless of the surface property. However, AuNPs displayed significant surface charge-dependent effects on important biochemical parameters such as the concentrations of hydrogen peroxide in plant roots and the activities of several enzymatic antioxidants. Deposition of AuNPs on plant root surface was observed for all types of AuNPs. However, surface charge affected the internalization of AuNPs in plant root cells and their subcellular localization. Overall, the study revealed that the physical and chemical properties AuNPs play important roles in their interactions with agricultural crops and consideration of the unique properties of nanoparticles is important in assessing their food safety concerns.
\end{abstract}

\section{Keywords}

Gold nanoparticles, Surface charge, Phytotoxicity, Subcellular localization, Phaseolus vulgaris

\section{Introduction}

Engineered nanoparticles (ENPs) of different shapes and sizes $(1-100 \mathrm{~nm}$ ) are increasingly incorporated into manufactured products. Recently, the unique properties of gold nanoparticles (AuNPs) have attracted tremendous interest in scientific research and industrial applications such as optical sensors, targeted drug delivery carriers, and cancer therapy agents [1-6]. A major effort with the application of AuNPs is to enhance the biocompatibility of AuNPs through surface modification with different coating materials [7, 8]. Consequently, AuNPs with various surface modifications and properties are fully expected in the environment.

Most previous toxicity studies with AuNPs concentrated on the potential cytotoxicity of AuNPs to different cell lines or model organisms, with a few limited studies focused on plant AuNPs interactions. These previous studies indicated that AuNPs could be taken up by plant roots and accumulated in plant tissues and the extent of plant uptake depended upon the unique properties of AuNPs $[9,10]$. It has been reported that the cytotoxicity of AuNPs is dependent 
on the NPs size instead of the functionalized surface chemistry [11]. However, a separate study with molecular simulation suggested that AuNP surface charge and the charge density play a key role in their penetration and disruption of lipid membranes [12]. Several previous studies on the phytotoxicity and uptake of AuNPs by plants have also demonstrated the strong effect of surface properties. For example, Judy et al. [13] examined the accumulation of 10,30 , and $50 \mathrm{~nm}$ tannate and citrate coated AuNPs by two plant species: a monocot wheat and a dicot tobacco and reported that tobacco plants showed higher accumulation of $50 \mathrm{~nm}$ citrate coated AuNPs than smaller citrate coated AuNPs, while the accumulation of $50 \mathrm{~nm}$ tannate coated AuNPs was less than the smaller AuNPs with the same coating. The authors also reported the detection of AuNPs with both coating materials in tobacco but not in wheat, indicating that the uptake and accumulation of AuNPs was also species dependent.

Koelmel et al. [14] investigated the uptake and spatial distribution of $2 \mathrm{~nm}$ (core diameter) AuNPs functionalized with cationic thioalkylated tetraethylene glycol ligand (TTMA; +), neutral tetraethylene glycol (TEGOH, o), and anionic tetraethylene glycol acetic acid ligand (TEGCOOH, -) coatings to rice plants under hydroponic systems. The study found that the bioaccumulation of AuNPs in root and shoot tissues of rice plants are surface charge dependent at treated concentrations and duration as determined by a laser ablation inductively coupled plasma mass spectrometry (LAICP-MS). After five days of treatment, the concentration of AuNPs with positive surface charge was the highest in plant root tissues, followed by neutral and then negatively charged AuNPs. In the shoot tissues of treated rice plants, however, the order was reversed [14]. The results agreed well with another study which demonstrated that positively charged AuNPs were more accumulated in plant roots, but negatively charged AuNPs displayed highest root to shoot translocation, followed by neutrally charged AuNPs [15]. Clearly, the literature suggested that the surface properties of AuNPs play a critical role in their uptake and accumulation in plants. However, despite these progresses, detailed evaluation on the impact of surface charge of AuNPs and other metallic NPs on their phytotoxicity, accumulation and subcellular localization and their impact on plant physiological and biochemical processes is still rare.

The objective of this study was to investigate the uptake and subcellular localization of AuNPs with different surface coatings/charges and their potential toxicity to Phaseolus vulgaris (bush bean). Bush bean is a dicotyledonous plant and is a common variety bean crop with a rich cultivation history that is commonly grown worldwide for its edible seed [16]. It also holds significant agricultural importance as a staple vegetable and as an animal feeding stock.

\section{Materials and Methods}

\section{Chemicals}

Uncoated gold nanoparticle (UN-AuNPs) dispersion $(14 \mathrm{~nm})$ was purchased from US Research Nanomaterials, Inc., (Houston, TX, USA). AuNPs suspensions (20 nm) coated with branched polyethyleneimine (bPEI-), polyethylene glycol (PEG-), and citrate (Cit-) were purchased from Nanocomposix (San Diego, CA, USA). Quarter strength Hoagland solution was prepared by dissolving modified Hoagland basal salt mixture purchased from PhytoTechnology Laboratories (Lenexa, KS, USA) in deionized (DI) water.

\section{Characterization of gold nanoparticles}

The primary NP size and zeta potential of coated AuNPs were provided by the manufacturer and are listed in Table 1. According to the manufacturer, the particle size was determined using a JEOL 1010 transmission electron microscope (TEM) (JEOL USA, Inc., MA) and the zeta potential and hydrodynamic sizes were determined with a Malvern Zetasizer Nano ZS instrument (Malvern Instrument Ltd, Worcestershire, UK). At the end of the experiment, the size and morphology of AuNPs were determined again using a Hitachi H-7650 TEM (Hitachi High-Technologies Corporation, Tokyo, Japan). Average particle size for each treatment was determined from the TEM images for at least 200 individual particles using ImageJ software (NIH Bethesda, MD, USA). The hydrodynamic size and zeta potential of

Table 1: Physical and chemical properties of AuNPs with different surface coatings.

The reported values are presented as mean \pm standard deviation $(n=3) .{ }^{*}$ Data are obtained from the manufacturers. a, b refers two different vendors. ${ }^{1}$ Data were measured with freshly prepared solutions without exposure to plant material. ${ }^{2}$ Data were measured from solutions exposed to plants for five days (after experiment termination).

\begin{tabular}{|c|c|c|c|c|c|c|c|c|}
\hline \multirow[t]{4}{*}{$\begin{array}{c}\text { Gold } \\
\text { Nanoparticles }\end{array}$} & \multicolumn{2}{|c|}{ Primary Particle Size } & \multicolumn{3}{|c|}{ Hydrodynamic Size } & \multicolumn{3}{|c|}{ Zeta Potential } \\
\hline & {$\left[1000^{a} / 50^{b}\right.$} & [5 ppm] & [50 ppm] & \multicolumn{2}{|c|}{ [5 ppm] } & [50 ppm] & \multicolumn{2}{|c|}{ [5 ppm] } \\
\hline & \multicolumn{2}{|c|}{$(\mathbf{n m})$} & \multicolumn{3}{|c|}{$(\mathbf{n m})$} & \multicolumn{3}{|c|}{$(\mathrm{mV})$} \\
\hline & Manufacturer" & {$[\text { End }]^{2}$} & Manufacture & ${\text { [Initial }]^{1}}$ & {$[\text { End }]^{2}$} & Manufacture & ${\text { [Initial }]^{1}}^{1}$ & {$[\text { End }]^{2}$} \\
\hline Uncoated & $14^{\mathrm{a}}$ & $12.67 \pm 7.48$ & n.d. & $87.47 \pm 10.98$ & $155.33 \pm 29.82$ & n.d. & $-10.42 \pm 1.24$ & $-20.10 \pm 4.18$ \\
\hline bPEI & $18.10 \pm 1.90^{b}$ & $19.56 \pm 2.50$ & 39.5 & $1535.33 \pm 215.51$ & $1346.67 \pm 129.21$ & 29.1 & $5.35 \pm 0.19$ & $-22.83 \pm 2.74$ \\
\hline PEG & $19.60 \pm 1.90^{b}$ & $20.54 \pm 2.22$ & 47.0 & $22.77 \pm 1.01$ & $306.30 \pm 21.41$ & -11.2 & $-15.47 \pm 1.70$ & $-28.00 \pm 8.64$ \\
\hline Citrate & $18.70 \pm 1.70^{b}$ & $19.69 \pm 2.65$ & 21.7 & $24.34 \pm 1.66$ & $205.20 \pm 14.31$ & -40.0 & $-19.17 \pm 1.16$ & $-29.03 \pm 3.23$ \\
\hline
\end{tabular}


AuNPs were determined using a dynamic light scattering (DLS) instrument (Zetasizer Nano Z90 Malvern Instrument Ltd, Worcestershire, UK) in both the freshly prepared solution and the solutions at the end of the experiment after plant exposure. Solutions were shaken vigorously by hand before DLS measurements.

\section{Plant growth}

Phaseolus vulgaris (bush bean) seeds were obtained from Johnny's Selected Seeds (Winslow, ME, USA). The seeds were first surface sterilized with $15 \%$ hypochlorite solution $(\mathrm{NaClO})$ for 10 minutes, and then successively rinsed with DI water for at least three times. The sterilized seeds were germinated in a petri dish for seven days on a filter paper moistened with quarter strength Hoagland solution. After germination, healthy seedlings were transferred to $50 \mathrm{~mL}$ plastic centrifuge tubes filled with quarter strength Hoagland solution. The seedlings were allowed to grow in a growth cart with a $16 \mathrm{hrs}$.-light/ $8 \mathrm{hrs}$.-dark cycle for another seven days at room temperature $\left(21-24{ }^{\circ} \mathrm{C}\right)$. The light intensity in the growth cart was around $133 \mu \mathrm{mol} \mathrm{m} \mathrm{m}^{-2} \mathrm{~s}^{-1}$. Following the incubation period, the seedling roots were thoroughly rinsed with DI water to remove Hoagland solution residues and the liquid in the test tubes were replaced with solutions containing $5 \mathrm{mg} / \mathrm{L}$ of UN-, bPEI-, PEG-, and Cit-AuNPs or just tap water (control) for five days. The solutions were replenished every other day at constant volume for treatment groups. Tap water was used so that the plants were not completely depleted of nutrients. Eight replicates were prepared in each treatment scenario.

\section{Plant health monitoring}

Multiple parameters indicative of plant physiological health was monitored. Plant seedling biomass was determined every other day and the relative biomass increase was calculated using the following equation $\left[\left(\mathrm{m}_{\mathrm{i}}-\mathrm{m}_{\mathrm{o}}\right) / \mathrm{m}_{\mathrm{o}}\right] \times 100(\%)$; where $\mathrm{m}_{\mathrm{o}}$ is the initial measurement and $\mathrm{m}_{\mathrm{i}}$ the successive measurement. Shoot elongation and root elongation were determined by measuring the tissue length with a ruler every other day and the elongation rate was computed by dividing the total elongation with days of treatment. Water transpiration was measured daily through the differences of water levels before replenishment.

\section{Chlorophyll content quantification}

To measure total chlorophyll content, $0.7 \mathrm{~g}$ fresh leaf tissues were randomly selected, excised and immersed in 15 $\mathrm{mL}$ of $95 \%$ acetone for $12 \mathrm{~h}$ at $4{ }^{\circ} \mathrm{C}$. Then, the absorbance of acetone solution containing chlorophyll was measured with a Varian CARY 50 Bio UV-Vis spectrophotometer at 663 and $645 \mathrm{~nm}$. The chlorophyll content was calculated using the equation reported previously [17].

\section{Hydrogen peroxide quantification}

Three replicates from each treatment were randomly selected for hydrogen peroxide $\left(\mathrm{H}_{2} \mathrm{O}_{2}\right)$ quantification following our previously established procedures [18]. Extraction of leaves and root tissues were performed separately. Leaves were cut from the plant seedlings and directly extracted without rinsing. The root tissues from each seedling were thoroughly rinsed with DI water to remove excess surface residues before extraction. Fresh biomass weighing $0.2 \mathrm{~g}$ were immediately ground in $2 \mathrm{~mL} 0.1 \%(\mathrm{v} / \mathrm{v})$ trichloroacetic acid (TCA) solution using a pestle and mortar on ice. The homogenate produced was then decanted into a $2 \mathrm{~mL}$ Eppendorf tube and centrifuged at $12,000 \mathrm{~g}$ for 15 minutes at $4^{\circ} \mathrm{C}$. Assay for $\mathrm{H}_{2} \mathrm{O}_{2}$ quantification were prepared as described by Velikova et al. [19]. The supernatant $(0.5 \mathrm{~mL})$ from the centrifuged homogenate was mixed with assay buffer containing $0.5 \mathrm{~mL}$ $10 \mathrm{mM}$ potassium phosphate $\left(\mathrm{KH}_{2} \mathrm{PO}_{4}\right)$ at $\mathrm{pH} 7$ and $1 \mathrm{~mL}$ of $1 \mathrm{M}$ of potassium iodide (KI) to a total volume of $2 \mathrm{~mL}$. The absorbance of the mixture was immediately measured using Varian CARY 50 Bio UV-Vis spectrophotometer at $390 \mathrm{~nm}$.

\section{Hydrogen peroxide visualization}

Visualization of the localization of $\mathrm{H}_{2} \mathrm{O}_{2}$ was performed as reported in our previous research [20]. For root tissues, fresh root sections of approximately four $\mathrm{cm}$ were excised and submerged in a jar containing $25 \mathrm{~mL}$ of $1 \mathrm{mg} / \mathrm{mL}$ of 3,3'-diaminobenzine (DAB) at $\mathrm{pH} 3.6$ for 8 hours. Stained roots were rinsed twice to remove residue $\mathrm{DAB}$ solution and then visualized using a Kruss MBL3000 light microscope equipped with 3-megapixel digital camera. In the case of leaf tissues, excised leaves were also immediately submerged in DAB solution as described above for eight hours. Stained leaves were then transferred into a new jar containing absolute ethanol (200 proof) and boiled for approximately 8 minutes to remove chlorophyll before they were observed under the microscope.

\section{Estimation of antioxidant enzymes activity}

Activities of interested antioxidant enzymes were carried out as follows. $0.2 \mathrm{~g}$ of fresh leaf or root tissues was randomly selected from three independent replicates from each treatment group. No rinsing was performed on leaf tissues, while the root tissues were thoroughly rinsed for at least three times with DI water to clear the root surface. Fresh biomass was snap frozen in liquid nitrogen, and ground in $100 \mathrm{mM} \mathrm{KH_{2 }} \mathrm{PO}_{4}$ buffer ( $\mathrm{pH}$ 7.0) containing $0.1 \mathrm{mM}$ ethylenediaminetetraacetic acid (EDTA) and $0.1 \%(\mathrm{w} / \mathrm{v})$ of polyvinylpolypyrrolidone (PVPP) using a pestle and mortar on ice. Homogenate were then decanted into a $2 \mathrm{~mL}$ Eppendorf tube and centrifuged at $10,000 \mathrm{~g}$ for 20 minutes at $4{ }^{\circ} \mathrm{C}$. Resulting supernatant were then used to estimate the activities of antioxidant enzymes: superoxide dismutase (SOD), guaiacol peroxidase (GPX) and catalase (CAT).

Estimation of SOD (EC1.15.1.1) was performed as described by Kakkar et al. [21]. SOD activity was determined from a $2 \mathrm{~mL}$ assay buffer containing $200 \mu \mathrm{L}$ of supernatant mixed with $1.2 \mathrm{~mL}$ of $25 \mathrm{mM}$ sodium pyrophosphate $\left(\mathrm{Na}_{2} \mathrm{HPO}_{4}\right)$ at $\mathrm{pH} 8.3,100 \mu \mathrm{L}$ of $186 \mu \mathrm{M}$ of phenazine methosulphate (PMS), $300 \mu \mathrm{L}$ of $300 \mu \mathrm{M}$ of nitro blue tetrazolium (NBT) and $200 \mu \mathrm{L}$ of $780 \mu \mathrm{M}$ of nicotinamide adenine dinucleotide (NADH). The absorbance of the $2 \mathrm{~mL}$ reagent mixture was continuously measured at 10 -second intervals for 60 seconds using a Varian CARY 50 Bio UV-Vis spectrophotometer (Agilent Technologies, Santa Carla, CA, USA) at $560 \mathrm{~nm}$. One unit of SOD is defined as the required amount of SOD that contributes $50 \%$ inhibition of NBT 
reduction. NBT reduction in 60 seconds without supernatant extract was also performed as blank control. SOD activity is expressed as Unit/mg total protein.

Estimation of GPX (EC1.11.1.7), and CAT (EC1.11.1.6) activities were performed following methods described by Maehly and Chance [22]. GPX assay was employed based on the formation of tetraguaiacol through the oxidation of guaiacol. The oxidation kinetics of guaiacol was measured in a $2 \mathrm{~mL}$ buffer containing $50 \mu \mathrm{L}$ of supernatant, $1.95 \mathrm{~mL}$ of $100 \mathrm{mM} \mathrm{KH} \mathrm{PO}_{4}$ buffer at $\mathrm{pH} 7.0$ containing $0.1 \mathrm{mM}$ of EDTA, $2 \mu \mathrm{L}$ of $10 \mathrm{mM}$ guaiacol and $5 \mu \mathrm{L}$ of $10 \mathrm{mM} \mathrm{H}_{2} \mathrm{O}_{2}$ at $470 \mathrm{~nm}$ wavelength for 60 seconds at 10 seconds intervals using a Varian CARY 50 Bio UV-Vis spectrophotometer. The GPX activity was determined as $\mu \mathrm{mol}$ tetraguaiacol formed/ $\mathrm{mg}$ protein/min.

CAT assay was implemented based on $\mathrm{H}_{2} \mathrm{O}_{2}$ reduction catalyzed by CAT that results in a decreased absorbance at $240 \mathrm{~nm}$. The estimation of CAT activity was determined from a $1 \mathrm{~mL}$ assay buffer containing $15 \mu \mathrm{L}$ of supernatant, $982.5 \mu \mathrm{L}$

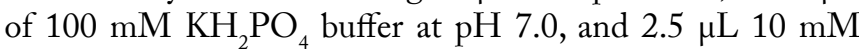
$\mathrm{H}_{2} \mathrm{O}_{2}$. The continuous decomposition of $\mathrm{H}_{2} \mathrm{O}_{2}$ was measured using a Varian CARY 50 Bio UV-Vis spectrophotometer at $240 \mathrm{~nm}$ at 10 -second intervals for one minute to determined CAT activity. A linear regression analysis was performed to determine the reaction constant of decreasing absorbance based on the slope of measured readings. The CAT activity was determined as $\mu \mathrm{mol} / \mathrm{mg}$ protein $/ \mathrm{min}$.

\section{Estimation of total protein content}

Total protein content measurements were determined using $100 \mu \mathrm{L}$ of the supernatant extract described above. Total protein content was determined using a non-interfering protein assay extraction kit (Geno Technology, St. Louis, MO, USA) carried out following the manufacturer's instructions.

\section{Scanning electron microscopy (SEM) with $x$-ray} microanalysis of Au on root surface

Fresh bush bean root tissues of control and treated groups at day 5 were randomly selected and excised with a scalpel. At least three root tissues per group were excised and placed onto one SEM pin stub with a conductive carbon tab. The prepared samples were then immediately placed onto the specimen mount inside the SEM chamber. No additional preparation was performed on mounted samples. SEM micrographs of bush bean root tissue were captured using a FEI Quanta FEG450 SEM (Hillsboro, OR, USA) operated at $10 \mathrm{kV}$ under low vacuum mode with chamber vacuum pressure at $65 \mathrm{~Pa}$. Secondary electron (SE) and backscattered electron (BSE) micrographs were obtained by respective SE detector and directional backscatter detector. Energy dispersive X-Ray spectroscopy (EDS) analysis was performed using the attached $50 \mathrm{~mm}^{2}$ Oxford detector on the FEI Quanta FEG450 SEM operated at $30 \mathrm{kV}$.

\section{Transmission electron microscopy}

Fresh root tissues (2 $\mathrm{mm}$ in length) were randomly selected and excised. They were then placed into individual vials and pre-fixed with freshly prepared $2 \%$ glutaraldehyde in $0.05 \mathrm{M}$ Sorenson phosphate buffer ( $\mathrm{pH}$ 7.2) for two hours at room temperature. Following four Sorenson phosphate buffer rinses at intervals of 30 minutes, samples were post-fixed for 10 minutes in $2 \%$ osmium tetroxide. Three water rinses at 30 minutes intervals were subsequently performed. The samples were then serially dehydrated using 25\%, 50\%, 75\% ethanol for 20 minutes each and stored overnight at $4{ }^{\circ} \mathrm{C}$. Further dehydration continued with $100 \%$ ethanol for three times at 20 minute intervals. Samples were then infiltrated using LRW resin at room temperature (Electron Microscopy Sciences Hatfield, PA, USA) starting at 25\% LRW resin (v/v) in 100\% ethanol, 50\% LRW resin (v/v) in 100\% ethanol, and 75\% LRW resin (v/v) in $100 \%$ ethanol for two cycles at one-day intervals for each incremental increase. Last infiltration process requires $100 \%$ LRW resin for four cycles at one-day interval at room temperature. Anaerobic polymerization of LRW resin starts with the transfer of root tissues from vials into individual gelatin embedding capsules (Electron Microscopy Sciences Hatfield, PA, USA) and left to cure in an oven at $60{ }^{\circ} \mathrm{C}$ for 72 hours. Ultrathin sections ( 50 to $70 \mathrm{~nm}$ ) with gold reflections were cut using a diamond knife attached on a Leica EM UC6 ultramicrotome (Leica Microsystems, Austria). Sections were then collected on a 200-mesh copper grids stained with $2 \%$ uranyl acetate and lead citrate. Ultrastructure of bush bean root tissues were observed and photographed using a Hitachi $\mathrm{H}-7650 \mathrm{TEM}$ at $60 \mathrm{kV}$ to examine subcellular localization of AuNPs.

\section{Data analysis}

Data are expressed as mean \pm standard deviation; unless otherwise indicated. One-way ANOVA analysis was performed and $p<0.05$ was considered as significant. Tukey's HSD method was conducted as post hoc comparison to identify differences between treatments. All statistical tests were processed with SPSS Statistics Version 20 (SPSS Inc., Chicago, IL, USA).

\section{Results and Discussion}

\section{Gold nanoparticle characterization}

TEM micrographs and size distributions of AuNP solutions exposed to bush bean seedlings for five days are shown in Figure 1. The mean particle sizes were $12.67 \pm$ $7.48,19.56 \pm 2.50,20.54 \pm 2.22$, and $19.69 \pm 2.65 \mathrm{~nm}$ for UN-, bPEI-, PEG-, and Cit-coated AuNPs respectively. For UN-AuNPs, about $60 \%$ of NPs were in the range of 4 to 12 $\mathrm{nm}$, while only $25 \%$ were in the range of 12 to $20 \mathrm{~nm}$. The results suggested that UN-AuNPs were slightly smaller than the reported values by the manufacturer (Table 1). Surface coated AuNPs (bPEI-, PEG-, and Cit-) were comparable to the reported size range after five days. The majority of AuNPs was found to be roughly spherical, with some particles having irregular, triangular, or oval shapes (Figure 1). Hydrodynamic size and zeta potential of AuNPs are provided in Table 1. DLS measurements showed that the mean hydrodynamic diameters of freshly prepared AuNPs solutions were 87.47 $\pm 10.98,1535.33 \pm 215.51,22.77 \pm 1.01,24.34 \pm 1.66 \mathrm{~nm}$ for UN-, bPEI-, PEG-, and Cit-AuNPs; respectively. After five days of exposure to plants, their mean hydrodynamic diameters increased to $155.33 \pm 29.8,1346.67 \pm 129.2,306.30$ 


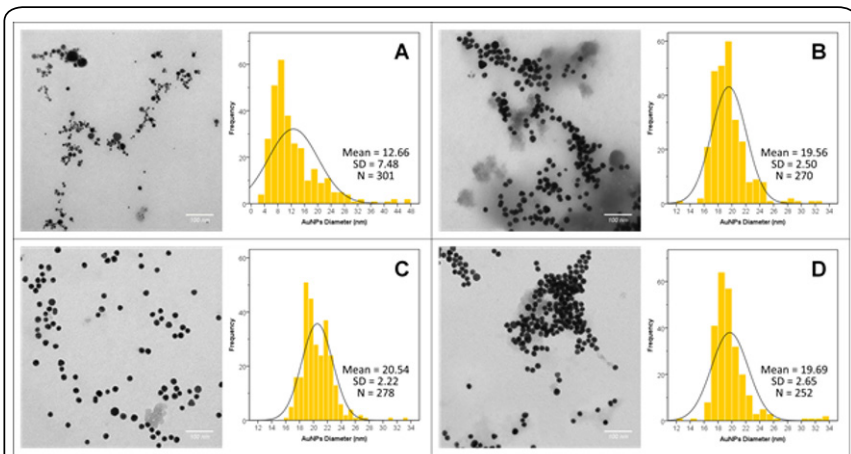

Figure 1: Particle size analysis of AuNPs after treatment period at day 5. Panel shows TEM micrograph of AuNPs and particle size distribution (A) Uncoated, (B) bPEI-coated, (C) PEG-coated, and (D) Citrate-coated. Scale bar shows $100 \mathrm{~nm}$.

$\pm 21.4,205.20 \pm 14.31 \mathrm{~nm}$ respectively. The results indicated substantial aggregation of bPEI-AuNPs immediately after dilution in water. After five days, the hydrodynamic size of AuNPs all became larger except for bPEI-AuNPs, which still had the largest AuNPs hydrodynamic size. The size differences between different AuNPs were consistent with the color differences of AuNPs solutions (Supplementary Figure 1), which depend on the AuNP sizes of the dispersions [23]. Zeta potential of the AuNPs as reported by the manufactures are shown in Table 1. Different coating materials were added to AuNPs surfaces to enhance the NP stability and bestow the NPs different surface charges. However, zeta potential measurements of AuNPs in freshly prepared solutions indicated that the zeta potential for all NPs have mitigated by the solution and their zeta potential in the freshly prepared solutions were $-10.42 \pm 1.24,+5.35 \pm 0.19,-15.47 \pm 1.70$, $-19.17 \pm 1.16 \mathrm{mV}$ for UN-, bPEI-, PEG- and Cit-AuNPs; respectively. After five days, zeta potential turned negative for all AuNPs ranging from -20.10 to $-29.03 \mathrm{mV}$ [Table 1]. The similar zeta potentials for all NPs after plants were grown in them for five days might be due to the adsorption of weak acids and enzymes excreted from plant roots on NP surface.

\section{Gold nanoparticle stability}

It is generally agreed that $\mathrm{Au}$ is one of the least chemically active metals that is resistant not only to heat and oxidative dissolution but also inert in strong alkalis and nearly all acid with the exception of selenic acid and nitro hydrochloric acid [24]. In a previous study, Zhai et al. [25] observed high uptake rates of both AuNPs and Au (III) ion by poplar cuttings. The authors also reported that AuNPs did not release $\mathrm{Au}$ ions in hydroponic exposure to poplar cuttings. Similarly, Zhu et al. [15] reported minimum dissolution of AuNPs with different coating materials based on the inductively coupled plasma mass spectrometry (ICP-MS) measurements of filtered and unfiltered AuNP solutions after five days of hydroponic exposed to radish. The authors reported that $>98 \%$ of AuNPs (filtered solution) were detected as intact particles and the amount of dissolved gold $(<5 \mathrm{pg} / \mathrm{mg})$ was insignificant compared with the original ( $2 \mathrm{ng} / \mathrm{mg}$ ) AuNPs (unfiltered solution) treatment concentration [15]. Consequently, it can be inferred that dissolution of AuNPs to Au ions was minimal in this study and the plant responses observed after their exposure to AuNPs were primarily induced by AuNPs rather than $\mathrm{Au}$ ions.

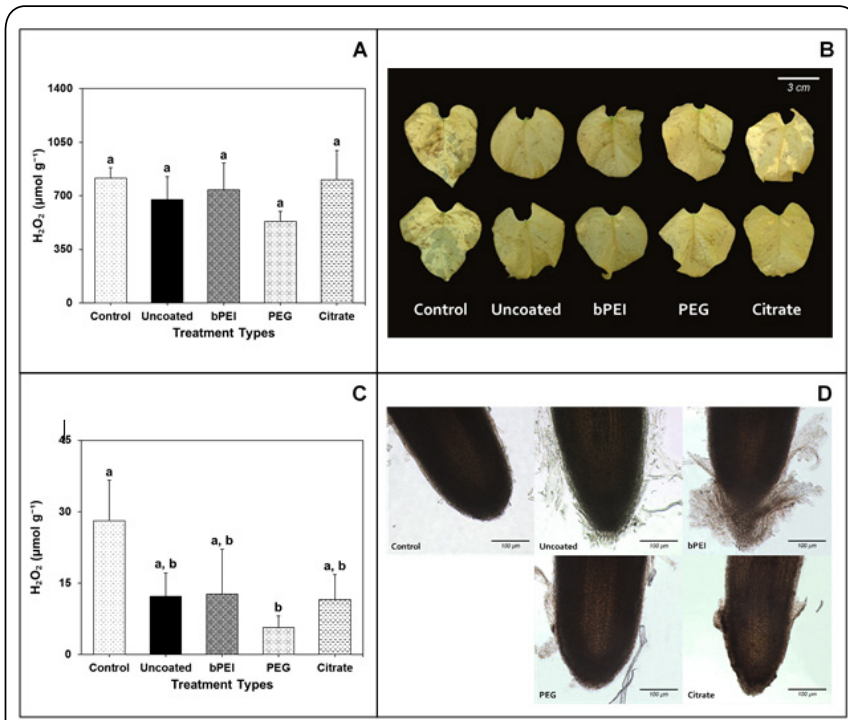

Figure 2: Hydrogen peroxide $\left(\mathrm{H}_{2} \mathrm{O}_{2}\right)$ accumulation in $(\mathrm{A})$ leaves and $(\mathbf{C})$ root tissues of bush bean seedlings exposed to AuNPs for 5 days. (B) Leaves and (D) roots stained for $\mathrm{H}_{2} \mathrm{O}_{2}$ using 3,3 diaminobenzene (DAB). The brown stains marked the localizations of $\mathrm{H}_{2} \mathrm{O}_{2}$ in leaves and root tissues. Reported values are average of three replicates for each treatment. Error bars represent standard deviation and letters above the error bars indicate the homogeneous subset grouping determined using one-way ANOVA analysis with post-hoc Tukey HSD test.
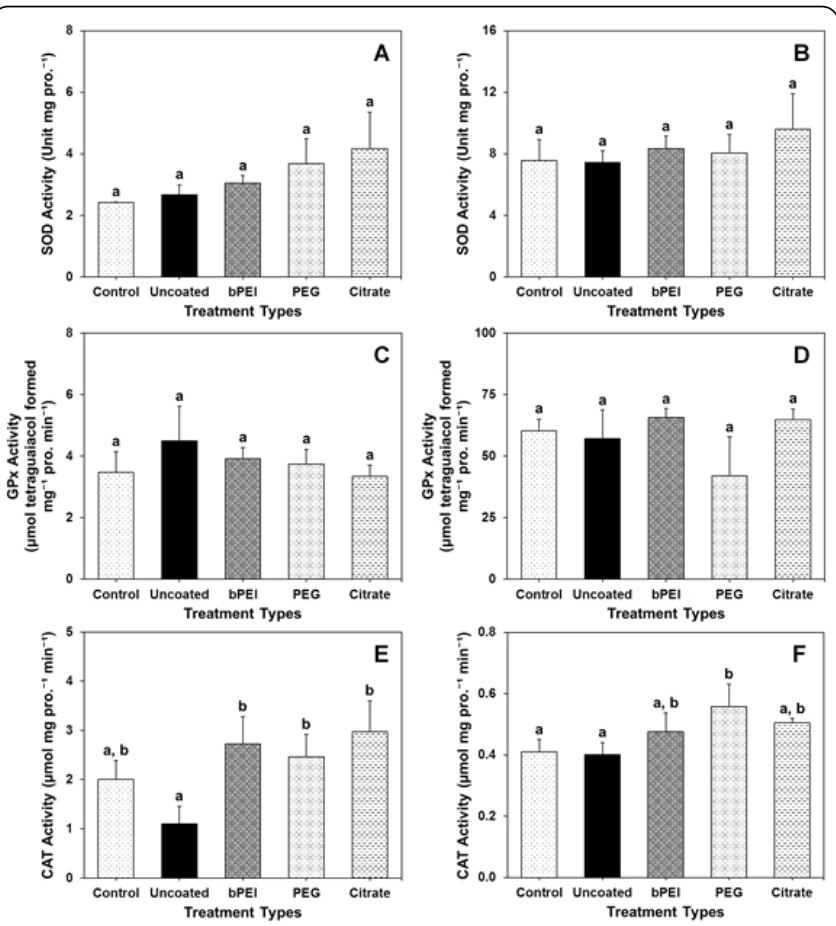

Figure 3: Superoxide dismutase (SOD), guaiacol peroxidase (GPX), catalase (CAT) activity in leaves $(\mathrm{A}, \mathrm{B}, \mathrm{C})$ and root tissues $(\mathrm{D}, \mathrm{E}, \mathrm{F})$ of bush bean seedlings exposed to AuNPs with different surface properties for five days. Reported values are the average of three replicates. Error bars represent standard deviation and letters above the error bars indicate the homogeneous subset grouping determined using one-way ANOVA analysis with post-hoc Tukey HSD test.

\section{Impact of AuNPs on plant growth}

Bush bean seedlings exposed to AuNPs with different surface properties were examined after five days of exposure. Measurements of plant biomass accumulation, transpiration, shoot and root elongation all indicated insignificant impact of 
AuNPs at $5 \mathrm{mg} / \mathrm{L}$, regardless of the surface coating properties (Supplementary Figure 2 and 3). Furthermore, no distinct morphological changes were observed for leaves or root structures of the treated bush bean seedling at the end of the experiment. Zhai et al. [25] also observed that (10, 25, and $50 \mathrm{~nm}$ ) bare AuNPs did not adversely affect poplar-cutting growth after six days of hydroponic exposure. Conversely, Arora et al. [26] demonstrated that cit-AuNPs (10 to $20 \mathrm{~nm}$ ) exposed through foliar spray to Brassica juncea improved plant growth and the enhancement was concentration dependent. $10 \mathrm{mg} / \mathrm{L}$ of AuNPs was found to be optimal for the growth of Brassica juncea [26]. However, their experiment duration was much longer than five days.

The total chlorophyll content obtained at the end of the five-day treatment is shown in Supplementary Figure 4. No significant differences were observed between treated groups and control. Chlorophylls are photosynthetic apparatus, which converts light energy into chemical energy such as glucose which the phototrophs can readily use for various activities such as cellular respiration. Reduced chlorophyll content could lead to impairment in light absorption [27, 28]. Measured total chlorophyll content in this study showed that AuNPs did not interrupt cellular activities important for photosynthesis. Interestingly, Arora and colleagues (2012) reported that citrate coated AuNPs (10 to $20 \mathrm{~nm}$ ) at $10 \mathrm{mg} / \mathrm{L}$ resulted in the highest chlorophyll content in Brassica juncea. Further increase in concentration $(25,50$, and $100 \mathrm{mg} / \mathrm{L})$ reduced the enhancive impact of AuNPs on chlorophyll content but they were still higher than control plants [26]. These results indicated that the approach of plant exposure to AuNPs (e.g. root exposure vs. foliar exposure) might be as important as the properties of AuNPs.

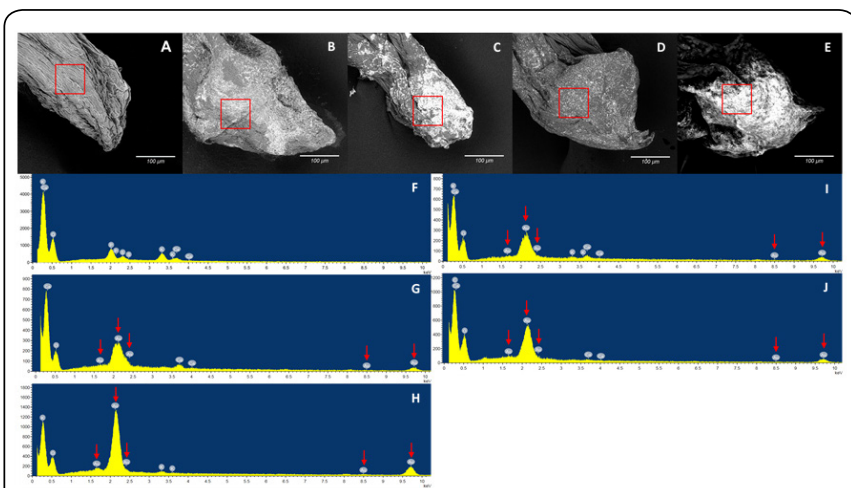

Figure 4: Scanning electron micrographs of bush bean root tips. Backscattered electron micrograph of (A) control, (B) uncoated, (C) bPEI, (D) PEG, and (E) Citrate coated AuNPs treated bush bean roots. $(\mathrm{E}),(\mathrm{F}),(\mathrm{G}),(\mathrm{H})$, and $(\mathrm{I})$ are spectrums of control, uncoated, bPEI, PEG, and Citrate coated AuNPs treated bush bean roots; respectively. Red box indicates the EDS analysis location and arrow indicates $\mathrm{Au}$ peaks. Scale bars show $100 \mu \mathrm{m}$.

Quantification and visualization of localized hydrogen peroxide $\left(\mathrm{H}_{2} \mathrm{O}_{2}\right)$

Unregulated increase of $\mathrm{H}_{2} \mathrm{O}_{2}$ in plant tissues could cause damage to nucleic acids, inhibit enzyme activities, even cause cell death [29]. $\mathrm{H}_{2} \mathrm{O}_{2}$ in plant tissues were visualized using 3,3'-diaminobenzidine (DAB) to determine their localization. For all treatments, visible brown precipitates were observed indicating the presence of $\mathrm{H}_{2} \mathrm{O}_{2}$ (Figure 2). $\mathrm{H}_{2} \mathrm{O}_{2}$ concentration in leaf tissues suggests that there were no significant differences between the control and the AuNPs treated plants (Figure 2). In the root tissues, however, PEG-AuNPs treatment led to significantly lower $\mathrm{H}_{2} \mathrm{O}_{2}$ than control plants (Figure 2). The results are consistent with an earlier study that $10 \mathrm{mg} / \mathrm{L}$ of citrate coated AuNPs resulted in insignificant differences in $\mathrm{H}_{2} \mathrm{O}_{2}$ in plants [13]. The authors did show that $\mathrm{H}_{2} \mathrm{O}_{2}$ content increased with increasing concentration of $\operatorname{AuNPs}^{2}(25,50$ and $100 \mathrm{mg} / \mathrm{L}$ ). Our results suggest that both surface coating and dosing concentration could affect the redox homeostasis of plants.

\section{Enzymatic antioxidant activity}

Essential oxidative stress indicators such as superoxide dismutase (SOD), guaiacol peroxidase (GPX), and catalase (CAT) are among the enzymatic antioxidants that regulate ROS levels in plants. The principle scavenger SOD starts the detoxification of ROS by dismutating superoxide radical $\mathrm{O}_{2}^{-}$into $\mathrm{H}_{2} \mathrm{O}_{2}$ and $\mathrm{O}_{2}$ [30]. Subsequently, GPX and CAT detoxify $\mathrm{H}_{2} \mathrm{O}_{2}$ into $\mathrm{H}_{2} \mathrm{O}$ and related by-products. In current study, SOD and GPX activities in shoot and root tissues were insignificantly different in comparison to those in control plants, regardless of the surface charges (Figure 3). The CAT activity, however, was significantly different between AuNPs treated plants and controls in both shoot and root tissues.

CAT activity in UN-AuNPs treated leaves was significantly lower than other plant leaves exposed to coated AuNPs but similar to control (Figure 3E). In root tissues, CAT activity in PEG-AuNPs treatment group was significantly higher than that of control and UN-AuNPs treatment groups but insignificantly different from other treatment groups (Figure $3 F)$. The results showed that properties of surface coatings had a greater effect on AuNPs interactions with roots than shoots, possibly due to the direct contact of these AuNPs with plant roots. Notably, the PEG-AuNPs resulted in significantly lower $\mathrm{H}_{2} \mathrm{O}_{2}$ accumulation and significantly higher CAT activity in root tissues. The results suggested that CAT was probably more predominant than GPX in detoxifying $\mathrm{H}_{2} \mathrm{O}_{2}$ levels in root tissues.

Scanning electron microscope (SEM) with $\mathrm{x}$-ray microanalysis of $\mathrm{Au}$ on root surface

AuNPs deposition was detected for all AuNPs treatments regardless of surface coatings (Figure 4). Backscattered electron micrographs reveal that, although all AuNPs were finely dispersed over a large area along the root tips surfaces, bPEI- and Cit-coatings were observed at more concentrated areas and also at higher intensity as compared to UN- and PEG-AuNPs. Energy Dispersive X-Ray Spectroscopy (EDS) analysis confirmed that the aggregates contained elemental $\mathrm{Au}$. EDS spectra is also shown in Figure 4. Control root tissues were also examined and deposition of elemental Au was not detected. The different patterns of deposition for PEG-AuNPs might derive from the neutral surface charge of primary nanoparticles. The lack of electrostatic repulsive force between the PEG-AuNPs probably allowed them to aggregate into relatively large aggregates rapidly once they were dispersed in liquid solution and precipitate out of the solution. 


\section{Transmission electron microscopy (TEM) of bush bean ultrastructure}

The ultrastructure of root tissues for all groups was examined near the quiescent center of the roots. Figure 5 displays TEM micrographs of control bush bean root tissues with healthy endodermis initial cells with large vacuoles. The nucleus with distinct nucleolus can be clearly seen in Figure 5 $\mathrm{A}_{2}$. Structurally normal vesicle (Figure $5 \mathrm{~A}_{2}$ ) and mitochondria (Figure $5 \mathrm{~A}_{1}$ ) are also observed. Figures $5 \mathrm{~A}_{3}$ and $5 \mathrm{~B}_{1}$ shows vacuoles of control root which was clear of AuNPs. Conversely, internalization and distribution of UNAuNPs can be seen in the cytoplasm (Figure $6 \mathrm{~A}_{1-3}$ and $6 \mathrm{~B}$ ), nucleus (Figure $6 \mathrm{~A}_{3}$ ), and adjacent region of the nucleolus (Figure $6 \mathrm{~B}_{1}$ ). Nucleus membrane with internalized UNAuNPs appears undamaged. Interestingly, bPEI-AuNPs was not observed inside plant root cells. A range of developing and developed root meristematic cells is shown near the quiescent center region (Figure 7A) and epidermal region (Figure 7B). Clear vacuoles and cytoplasm with no observed AuNPs is shown in Figure $7 \mathrm{~A}_{1-3}$. However, large aggregates of electron dense bPEI-AuNPs were identified outside of the root cells (Figure $7 \mathrm{~B}_{1}$ ). These figures further demonstrate that bPEIAuNPs did not internalize within bush bean but adhered to the epidermal surfaces of the primary or secondary roots. PEGAuNPs were observed in vacuoles (Figure $8 \mathrm{~A}, \mathrm{~A}_{2-3}$ and $8 \mathrm{~B}_{1}$ ), and vesicle (Figure $8 \mathrm{~A}_{1}$ ). Typical organelles such as mitochondria and vesicles can be seen without any morphological changes. In the case of Cit-coated treatment, several developing cells with electron dense material in the cytoplasm is shown in Figure 9A and 9B. Cit-AuNPs were detected to have internalized into cell wall and are located in the cytoplasm (Figure $9 \mathrm{~A}_{2-3}$ and $9 \mathrm{~B}_{1}$ ), and mitochondria (Figure $9 \mathrm{~A}_{1}$ ). In spite of the internalization, thick cell wall without membrane damage can be seen in in Figure $9 \mathrm{~A}$ and $9 \mathrm{~B}$.

Rico et al. [31] reported that ENPs can enter into plant cells via several possible pathways including binding to carrier proteins, through aquaporins, ion channels, endocytosis, or by binding to organic chemicals in the environmental media. Studies indicated that ENPs can enter into cells either

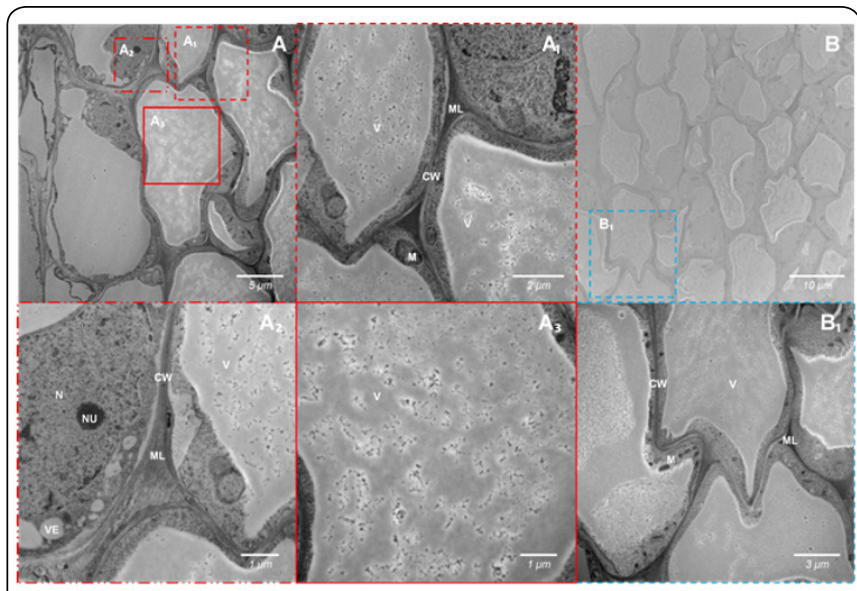

Figure 5: Transmission electron micrographs of control bush bean roots at day 5 after experiment termination. $A_{1}, A_{2}, A_{3}$, and $B_{1}$ are enlargements of $A$ and $\mathrm{B}$ at respective sites. Abbreviations: CW, Cell wall; ML, Middle lamella; $\mathrm{M}$, Mitochondria; N, Nucleus; NU, Nucleolus; V, Vacuole; VE, Vesicle. Scale bars of (A) $5 \mu \mathrm{m},\left(\mathbf{A}_{1}\right) 2 \mu \mathrm{m},\left(\mathbf{A}_{2}\right) 1 \mu \mathrm{m},\left(\mathbf{A}_{3}\right) 1 \mu \mathrm{m},(\mathbf{B}) 10 \mu \mathrm{m}$, and $\left(\mathbf{B}_{1}\right) 3 \mu \mathrm{m}$

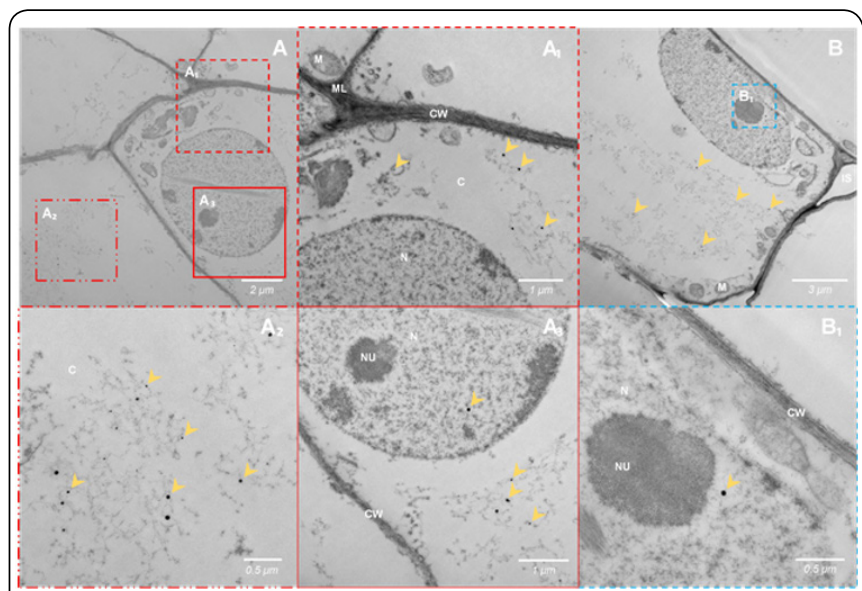

Figure 6: Transmission electron micrographs of bush bean root exposed to uncoated $A u N P$ s for 5 days. $A_{1}, A_{2}, A_{3}$, and $B_{1}$ are enlargements of $A$ and $B$ at respective sites. Abbreviations: $\mathrm{C}$, Cytoplasm; CW, Cell wall; IS, Intercellular space; ML, Middle lamella; M, Mitochondria; N, Nucleus; NU, Nucleolus. Arrows indicates nanoparticle(s). Scale bars of (A) $2 \mu \mathrm{m},\left(\mathbf{A}_{1}\right) 1 \mu \mathrm{m},\left(\mathbf{A}_{2}\right) 0.5$ $\mu \mathrm{m},\left(\mathbf{A}_{3}\right) 1 \mu \mathrm{m},(\mathbf{B}) 3 \mu \mathrm{m}$, and $\left(\mathbf{B}_{1}\right) 0.5 \mu \mathrm{m}$.

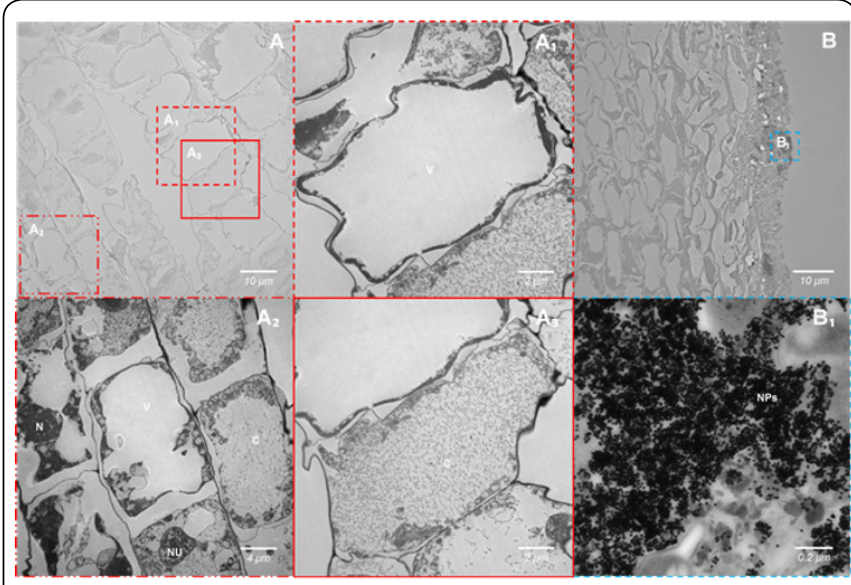

Figure 7: Transmission electron micrographs of bush bean root exposed to bPEI-coated AuNPs for 5 days. $A_{1}, A_{2}, A_{3}$, and $B_{1}$ are enlargements of $A$ and $B$ at respective sites. Abbreviations: $C$, Cytoplasm; N, Nucleus; NU, Nucleolus; $V$, Vacuole; NPs, Nanoparticles. Scale bars of (A) $10 \mu \mathrm{m},\left(A_{1}\right) 2 \mu \mathrm{m},\left(A_{2}\right) 4 \mu \mathrm{m}$, $\left(\mathbf{A}_{3}\right) 2 \mu \mathrm{m},(\mathbf{B}) 10 \mu \mathrm{m}$, and $\left(\mathbf{B}_{1}\right) 0.2 \mu \mathrm{m}$.

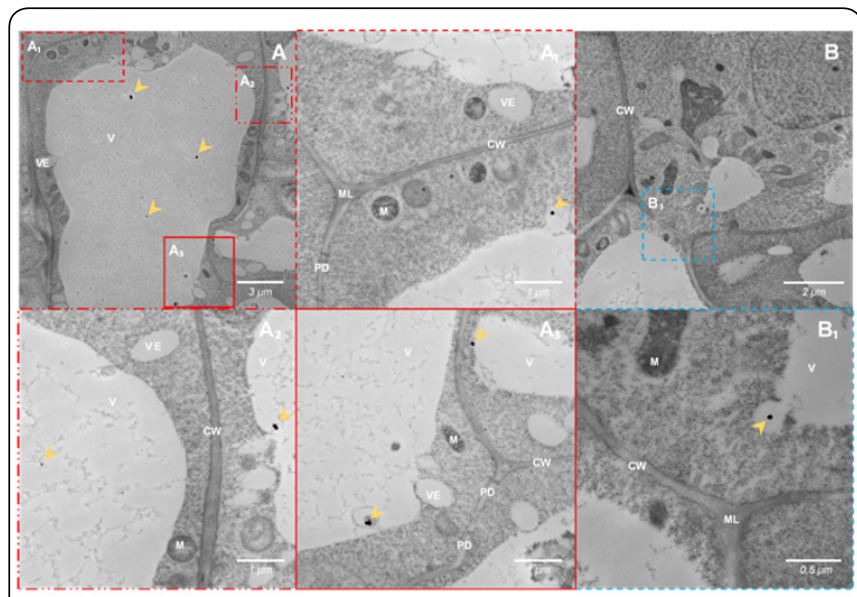

Figure 8: Transmission electron micrographs of bush bean root exposed to PEG-coated AuNPs for 5 days. $A_{1}, A_{2}, A_{3}$, and $B_{1}$ are enlargements of $A$ and $\mathrm{B}$ at respective sites. Abbreviations: $\mathrm{CW}$, Cell wall; ML, Middle lamella; M, Mitochondria; V, Vacuole; VE, Vesicle; PD, Plasmodesmata. Arrows indicates nanoparticle(s). Scale bars of (A) $3 \mu \mathrm{m},\left(\mathbf{A}_{1}\right) 1 \mu \mathrm{m},\left(\mathbf{A}_{2}\right) 1 \mu \mathrm{m},\left(\mathbf{A}_{3}\right) 1 \mu \mathrm{m},(\mathrm{B}) 2$ $\mu \mathrm{m}$, and $\left(\mathbf{B}_{1}\right) 0.5 \mu \mathrm{m}$ 


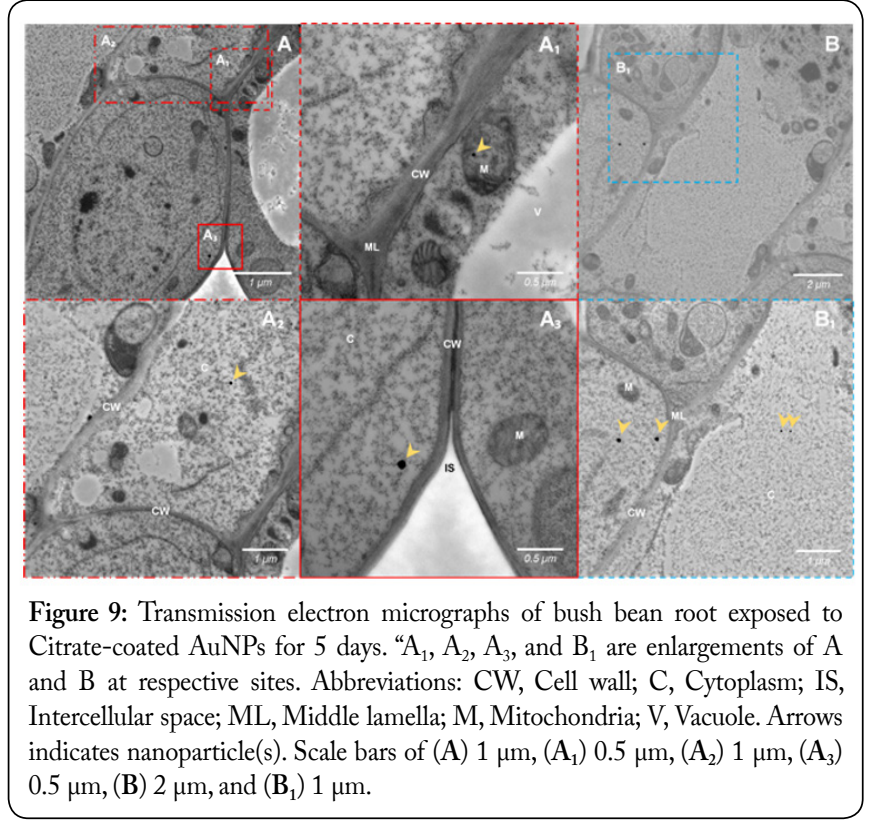

apoplastically or symplastically. Once ENPs are inside cells, ENPs may also be transported between neighboring cells through plasmodesmata [25]. It is generally agreed that root surface is negatively charged, and the uptake and distribution of ENPs are dependent on ENPs size, composition and surface chemistry. Zhu et al. [15] noticed that cationic AuNPs are more readily taken by plant roots, while anionic AuNPs were highly efficient in root-to-shoot translocation after hydroponic exposure for five days, consistent with the TEM observations in this study. Interestingly, other than bPEIAuNPs, all AuNPs displayed similar subcellular accumulation, possibly due to their similar size and surface charge after their exposure to the growing media. It has been anticipated that the bPEI-AuNPs would demonstrate the highest accumulation on root surface due to their positive charge. Indeed, dense accumulation of bPEI coated AuNPs was observed on root surface. In addition to the charge attraction, it appeared that the accumulation was also due to the deposition of relatively large aggregates as demonstrated by the large hydrodynamic size of bPEI-AuNPs. Such large aggregates would prohibit the entrance of bPEI coated AuNPs into root cells and this agreed well with the TEM analysis.

Once AuNPs entered into plant cells, they were frequently observed to distribute inside vacuoles and cytoplasm. In addition, some AuNPs were found to attach to different organelles. Specifically, organelle specific localization was observed within the nucleus of UN-AuNPs treatment, in vesicles of PEG-AuNPs treatment, and mitochondrion of Cit-AuNPs treatment. Interestingly, internalized AuNPs (UN-, PEG-, and Cit-) were not found in cell walls, middle lamella or intercellular spaces, in contrast with our previous studies with $\mathrm{AgNPs}$ [32] which were found to accumulate in the intercellular spaces of Arabidopsis root cells. AuNPs were also not found in plasmodesmata as in our previous studies for AgNPs or the study performed by Zhai et al. [25] for AuNPs in poplar roots. Reasons for the differences were unknown, but could be due to the surface chemistry of AuNPs treatment, plant species used and the exposure time. What is consistent with the previous studies was that some of the internalized AuNPs were also found to be aggregated to relatively larger sizes than their initial sizes. The aggregation might be due to the unique chemical environment in plant tissues. A key finding of this study was that AuNPs with different surface properties were found in different subcellular localizations and they were associated with different organelles. Because each organelle in plant cells carries out unique biochemical functions, it is understandable that AuNPs with different surface charge will result in different biochemical effects on plants which agrees well with our measurements on the biochemical responses of plants to AuNPs.

\section{Conclusion}

In closing, the study demonstrated that AuNPs with different coating materials and surface charges did not exhibit any significant differences in their impact on several important physiological parameters of plants at $5 \mathrm{mg} / \mathrm{L}$. However, their impact on the concentrations of $\mathrm{H}_{2} \mathrm{O}_{2}$ and CAT activity in plant root tissues were significantly different. AuNPs with different surface charge also showed different accumulation patterns on plant root surface and different internalization and subcellular localizations. Overall, the results indicated that surface property plays an important role in their impact on food crops and unique properties of ENPs must be considered in the assessment of the environmental fate and impact of ENPs.

\section{Acknowledgement}

The authors acknowledge the financial support of the USDA-AFRI (\#2011-67006-30181).

\section{References}

1. Cheng Y, C. Samia A, Meyers JD, Panagopoulos I, Fei B, et al. 2008. Highly efficient drug delivery with gold nanoparticle vectors for in vivo photodynamic therapy of cancer. J Am Chem Soc 130(32): 10643-10647. doi: 10.1021/ja801631c

2. Erathodiyil N, Ying JY. 2011. Functionalization of inorganic nanoparticles for bioimaging applications. Acc Chem Res 44(10): 925935. doi: 10.1021/ar2000327

3. Ghosh P, Han G, De M, Kim CK, Rotello VM. 2008. Gold nanoparticles in delivery applications. Advanced Drug Delivery Reviews 60(11): 1307-1315. doi: 10.1016/j.addr.2008.03.016

4. Huang X, El-Sayed IH, Qian W, El-Sayed MA. 2006. Cancer cell imaging and photothermal therapy in the near-infrared region by using gold nanorods. J Am Chem Soc 128(6): 2115-2120. doi: 10.1021/ ja057254a

5. Huang X, Jain PK, El-Sayed IH, El-Sayed MA. 2007. Gold nanoparticles: interesting optical properties and recent applications in cancer diagnostics and therapy. Nanomedicine 2(5): 681-693. doi: 10.2217/17435889.2.5.681

6. Peer D, Karp JM, Hong S, Farokhzad OC, Margalit R, et al. 2007. Nanocarriers as an emerging platform for cancer therapy. Nature Nanotechnology 2(12): 751-760. doi: 10.1038/nnano.2007.387

7. Mei BC, Susumu K, Medintz IL, Mattoussi H. 2009. Polyethylene glycol-based bidentate ligands to enhance quantum dot and gold nanoparticle stability in biological media. Nature Protocols 4(3): 412423. doi: $10.1038 /$ nprot.2008.243

8. Noh SM, Kim W-K, Kim SJ, Kim JM, Baek K-H, et al. 2007. Enhanced cellular delivery and transfection efficiency of plasmid DNA using 
positively charged biocompatible colloidal gold nanoparticles. Biochim Biophys Acta 1770(5): 747-752. doi: 10.1016/j.bbagen.2007.01.012

9. Dan Y,Zhang W,Xue R, Ma X, Stephan C, et al.2015. Characterization of gold nanoparticle uptake by tomato plants using enzymatic extraction followed by single-particle inductively coupled plasmamass spectrometry analysis. Environ Sci Technol 49(5): 3007-3014. doi: $10.1021 / \mathrm{es} 506179 \mathrm{e}$

10. Sabo-Attwood T, Unrine JM, Stone JW, Murphy CJ, Ghoshroy S, et al. 2012. Uptake, distribution and toxicity of gold nanoparticles in tobacco (Nicotiana xanthi) seedlings. Nanotoxicology 6(4): 353-360. doi: $10.3109 / 17435390.2011 .579631$

11. Pan Y, Neuss S, Leifert A, Fischler M, Wen F, et al. 2007. Sizedependent cytotoxicity of gold nanoparticles. Small 3(11): 1941-1949. doi: $10.1002 /$ smll.200700378

12. Lin J,Zhang H, Chen Z,Zheng Y.2010. Penetration of lipid membranes by gold nanoparticles: insights into cellular uptake, cytotoxicity, and their relationship. ACS Nano 4(9): 5421-5429. doi: 10.1021/nn1010792.

13. Judy JD, Unrine JM, Rao W, Wirick S, Bertsch PM. 2012. Bioavailability of gold nanomaterials to plants: importance of particle size and surface coating. Environ Sci Technol 46(15): 8467-8474. doi: 10.1021/es3019397

14. Koelmel J, Leland T, Wang H, Amarasiriwardena D, Xing B. 2013. Investigation of gold nanoparticles uptake and their tissue level distribution in rice plants by laser ablation-inductively coupled-mass spectrometry. Environmental Pollution 174: 222-228. doi: 10.1016/j. envpol.2012.11.026

15. Zhu Z-J, Wang H, Yan B, Zheng H, Jiang Y, et al. 2012. Effect of surface charge on the uptake and distribution of gold nanoparticles in four plant species. Environmental Science E Technology 46(22): 1239112398. doi: 10.1021/es301977w

16. Broughton WJ, Hernandez G, Blair M, Beebe S, Gepts P, et al. 2003 Beans (Phaseolus spp.)-model food legumes. Plant and Soil 252(1): 55128. doi: 10.1023/A:1024146710611

17. Reiss C. 1994. Experiments in plant physiology. Prentice Hall, New Jersey, USA.

18. Ma X, Wang Q, Rossi L, Zhang W. 2015. Cerium oxide nanoparticles and bulk cerium oxide lead to different physiological and biochemica responses in Brassica rapa. Environ Sci Technol. doi: 10.1021/acs. est.5b04111

19. Velikova V, Yordanov I, Edreva A. 2000. Oxidative stress and some antioxidant systems in acid rain-treated bean plants: protective role of exogenous polyamines. Plant Science 151(1): 59-66. doi: 10.1016/ S0168-9452(99)00197-1
20. Wang Q, Ebbs SD, Chen Y, Ma X. 2013. Trans-generational impact of cerium oxide nanoparticles on tomato plants. Metallomics 5(6): 753759. doi: $10.1039 / \mathrm{c} 3 \mathrm{mt} 00033 \mathrm{~h}$

21. Kakkar P, Das B, Viswanathan P. 1984. A modified spectrophotometric assay of superoxide dismutase. Indian J Biochem Biophys 21(2): 130-132.

22. Maehly A, Chance B. 1954. The assay of catalases and peroxidases. Methods Biochem Anal 1: 357-424.

23. Murphy CJ, Gole AM, Stone JW, Sisco PN, Alkilany AM, et al. 2008. Gold nanoparticles in biology: beyond toxicity to cellular imaging. Acc Chem Res 41(12): 1721-1730. doi: 10.1021/ar800035u

24. Merchant B. 1998. Gold, the noble metal and the paradoxes of its toxicology. Biologicals 26(1): 49-59. doi: 10.1006/biol.1997.0123

25. Zhai G,Walters KS, Peate DW, Alvarez PJ, Schnoor JL. 2014. Transport of gold nanoparticles through plasmodesmata and precipitation of gold ions in woody poplar. Environmental Science \& Technology Letters 1(2): 146-151. doi: $10.1021 / \mathrm{ez} 400202 \mathrm{~b}$

26. Arora S, Sharma P, Kumar S, Nayan R, Khanna P, et al. 2012. Goldnanoparticle induced enhancement in growth and seed yield of Brassica juncea. Plant Growth Regulation 66(3): 303-310. doi: 10.1007/s10725011-9649-z

27. Evans J, Poorter H. 2001. Photosynthetic acclimation of plants to growth irradiance: the relative importance of specific leaf area and nitrogen partitioning in maximizing carbon gain. Plant, Cell E Environment 24(8): 755-767. doi: 10.1046/j.1365-3040.2001.00724.x

28. Evans JR. 1996. Developmental constraints on photosynthesis: effects of light and nutrition. In: Baker NR (ed) Photosynthesis and the Environment. Advances in Photosynthesis and Respiration, Springer, Netherlands, pp 281-304. doi: 10.1007/0-306-48135-9_11

29. Sharma P, Jha AB, Dubey RS, Pessarakli M. 2012. Reactive oxygen species, oxidative damage, and antioxidative defense mechanism in plants under stressful conditions. Journal of Botany 2012: 217037. doi: $10.1155 / 2012 / 217037$

30. Apel K, Hirt H. 2004. Reactive oxygen species: metabolism, oxidative stress, and signal transduction. Annu Rev Plant Biol 55: 373-399. doi: 10.1146/annurev.arplant.55.031903.141701

31. Rico CM, Majumdar S, Duarte-Gardea M, Peralta-Videa JR, et al. 2011. Interaction of nanoparticles with edible plants and their possible implications in the food chain.J Agric Food Chem 59(8): 3485-3498. doi: 10.1021/jf104517j

32. Geisler-Lee J, Wang Q, Yao Y, Zhang W, Geisler M, et al. 2012. Phytotoxicity, accumulation and transport of silver nanoparticles by Arabidopsis thaliana. Nanotoxicology 7(3): 323-337. doi: $10.3109 / 17435390.2012 .658094$ 